\title{
Efectividad en el aprendizaje del proceso enfermero en el área hospitalaria
}

\author{
Rosa María Pecina Leyva \\ RESUMEN
}

El proceso enfermero (PE) permite fundamentar conocimientos teórico-prácticos que adquieren los alumnos durante su formación. Material y métodos: se realizó un estudio descriptivo, retrospectivo, y transversal. Con una muestra a conveniencia de 54 alumnos del tercer año de la carrera de licenciatura en enfermería. Se aplicó un cuestionario con una confiabilidad de 0.87. El análisis de los datos se realizó mediante la prueba de estadística descriptiva. Resultados: el 64\% de la muestra cuenta con un nivel de conocimientos suficientes sobre el PE y la media de calificación fue de 8.3 referente a la percepción de autoeficacia; en la aplicación del PE los alumnos refieren que se sienten seguros de aplicarlo ante situaciones reales según el 72\% y completamente seguros el 22\%. En el área hospitalaria el 67\% de los alumnos expresan que los docentes facilitaron el aprendizaje del PE, un 31\% opinan que los compañeros y un 2\% manifiesta que los profesionales de enfermería contribuyeron en la enseñanza del PE. Conclusiones: el nivel de conocimientos del alumno sobre el PE es suficiente, en relación a la percepción de autoeficacia para la aplicación del PE; los profesores si contribuyen en el aprendizaje de esta herramienta en el área hospitalaria, pero existen diversos factores que limitan su aplicación en los ámbitos hospitalarios, entre ellos se encuentran el exceso de pacientes y la falta de tiempo. Se deduce que la efectividad en el aprendizaje del PE aún no logra el efecto esperado en las áreas hospitalarias.

Palabras clave: efectividad, proceso enfermero, aprendizaje del alumno.

\section{Effectiveness in learning nursing process in a hospital area}

\begin{abstract}
The nursing process allows substantiating theoretical-practical knowledge acquired by students during their training in order to become nursing professionals. Therefore, a descriptive, retrospective, and transversal study was conducted, with a convenience sample of 54 students in the third year of their bachelor degree in nursing. A questionnaire was applied with a reliability of 0.87. The data analysis was performed using descriptive statistics. Results: $64 \%$ of the sample have sufficient level of knowledge about the nursing process, and the average score was 8.3. Perception of self-efficacy implementing nursing process, students report they feel safe applying it in the hospital area by $72 \%$, and $22 \%$ reported that they feel completely secure in its implementation. In hospital area, $67 \%$ of the students expressed that teachers facilitated the learning of nursing process, while $31 \%$ express that comrades facilitated this learning, and only $2 \%$ stated that nurses contribute to teaching nursing process. Conclusions: the level of knowledge in students is enough in regard to nursing process, and perception of self-efficacy implementing nursing process, students feel safe, they say that teachers help in learning this tool in hospitals, but several factors limit the application of nursing process in hospital settings, among this factors are: an excess in the number of patients, and the lack of time.
\end{abstract}

Key words: effectiveness, nursing process, student learning.

Correspondencia: Rosa María Pecina Leyva M.A. Universidad Autónoma de San Luis Potosí, S.L.P. México. Tel. 4448262324 Facultad de Enfermería E-mail: rosypecina@yahoo.com.mx 
$\mathrm{L}$

as escuelas y facultades en mayor o menor escala han iniciado esfuerzos con la característica especial, de integrar la educación a los procesos productivos y servicios ${ }^{1}$. Con el objetivo de lograr lo anterior en los últimos años las universidades de muchos países, se han encaminado a elevar la calidad de formación y superación de los recursos humanos ${ }^{1}$.

Los programas educativos en las áreas de enfermería contemplan la práctica como un espacio de aplicación de conocimientos, en el que el alumno aprende actividades profesionales, a través de la ejecución de acciones concretas en cuya realización se pretende que el alumno integre los conocimientos teóricos.

Para la formación de profesionales de enfermería se cuenta con un método científico que permite fundamentar los conocimientos teórico-prácticos que adquieren los alumnos durante su formación, esta herramienta fundamental se llama proceso enfermero (PE) y con ella los profesionales se incorporan a una nueva dimensión en la actividad de cuidar.

Aplicar el PE, nos permitirá identificar la trascendencia de los contenidos teóricos o técnico-instrumentales en la profesión de enfermería para proporcionar cuidado de calidad. Por lo tanto; la aplicación de este método científico en la práctica asistencial enfermera, permite prestar cuidados de una forma racional, lógica y sistemática, Iyer expresa que la aplicación del proceso del cuidado es útil en la práctica enfermera porque tiene repercusiones en 3 niveles: a nivel profesional sobre el cuidado del paciente y sobre la profesión ${ }^{2}$.

Para la Asociación Americana de Enfermería el proceso es considerado como estándar para la práctica de esta profesión, su importancia ha exigido cambios sustanciales en sus etapas favoreciendo el desarrollo de la enfermería como disciplina científica y ha incrementado la calidad en la atención del paciente, familia y comunidad ${ }^{2}$.

\section{OBJETIVO}

El presente estudio tiene como propósito determinar los factores que influyen en la efectividad del aprendizaje del PE en las áreas hospitalarias, con el fin de resolver las dificultades que los alumnos presentan en esta herramienta metodológica, para plantear estrategias tanto en el ámbito educativo como en el asistencial, y así elevar la calidad de los cuidados de enfermería.

\section{MARCO TEÓRICO}

La educación es parte importante de las actividades de aprendizaje que se realizan comúnmente. Los retos de la educación superior para el Siglo XXI; plantean la necesidad de un nuevo proceso educativo fundamentado en los principios de excelencia, calidad y pertinencia ${ }^{1}$.

Este proceso ha sido vinculado directamente a cambios políticos, económicos y sociales; que se han generado en los diferentes países donde el desarrollo social de la ciencia, técnica, práctica e investigación han obligado a aplicarlo no tan sólo en el discurso, sino en la práctica efectiva, en donde están visibles los conceptos de eficiencia, calidad y exigencia en los procesos educativos que realizan las universidades cada vez más comprometidas y en interacción con la sociedad ${ }^{1}$.

Ramón S. Salas; define cuatro conceptos diferentes de la calidad. 1. Excepcional el que ha sido el concepto académico tradicional. 2. Se fundamenta en la relación de la consistencia de un producto que es la conceptualización propia de la producción industrial en el cumplimiento de una misión en el concepto gerencial. 3. Es aquel donde se enmarca el análisis de eficiencia y eficacia del cumplimiento de una tarea o misión en la satisfacción de las necesidades del usuario. 4. Es la transformación del hombre en el concepto educacional o pedagógico contemporáneo de formación de los individuos y grupos sociales ${ }^{1}$.

De esta manera la calidad pasó a ser un elemento esencial, que sirve para evaluar eficiencia, efectividad y eficacia de los servicios.

Stephen Covey define la efectividad como el equilibrio entre eficacia y eficiencia, entre producción y capacidad de producción. El término efectividad es la capacidad de lograr un efecto deseado, esperado o anhelado ${ }^{3}$.

La eficacia para Koontz y Weihrich es el cumplimiento de objetivos ${ }^{4}$, y para Robbins y Coulter; es hacer las cosas correctas, es decir las actividades de trabajo con las que la organización alcanza sus objetivos ${ }^{5}$.

Para el presente estudio la conceptualización de la efectividad pedagógica hace referencia al hecho de como aprende el alumno, el papel del docente en el manejo de los contenidos incluídos en la propuesta didáctica en el currículo y la relación enseñanza-aprendizaje. Es decir; tiene relación con la articulación teórica con las instancias prácticas.

La educación en enfermería es importante para evaluar la eficacia del aprendizaje, conocer si lo planteado dentro del currículo enfermero y los programas educativos, en enfermería que lo operacionalizan, logran los objetivos educativos, de ahí la importancia de contar con elementos o instrumentos de evaluación que evidencien si son efectivos o no en el aprendizaje del alumno. 
En concecuencia, el nivel de aprendizaje se observa desde diferentes aspectos que favorecen a los alumnos para que puedan explicar los conceptos y aplicarlos, o bien relacionarlos entre sí de diferentes formas donde puedan retroalimentar lo aprendido.

La enfermería requiere de aplicación del conocimiento y habilidades específicas de la disciplina enfermera porque nos permitirá identificar claramente el objeto de acción de enfermería, así como competencias y obligaciones en la atención del paciente.

El PE adquiere cada día mayor relevancia en la formación de enfermeras (os), porque es un método que facilita la ejecución de los cuidados de enfermería.

Actualmente; existe gran cantidad de literatura sobre el PE que comprueba su efectividad para proporcionar asistencia de calidad e investigaciones que se publican continuamente y que se han realizado en diferentes ámbitos. A su vez existe abundante literatura de investigación sobre el éxito del PE en el cuidado de pacientes en diferentes etapas del ciclo vital, con diferentes patologías y donde se refleja exitosamente la aplicación de esta herramienta metodológica.

Aplicar el PE en el cuidado enfermero, nos permite identificar la trascendencia de los contenidos teóricos o técnicoinstrumentales en la profesión de enfermería para proporcionar cuidado de calidad. En México algunas investigaciones apoyan la importancia del PE en la profesión de enfermería.

$\mathrm{Al}$ respecto la educación en enfermería en México y el PE parecieran un contenido común en las escuelas y Facultades de Enfermería; pero no es así, pueden enseñarlo pero no se aplican en la práctica clínica Hdz V expresa que la formación de Enfermería en México, como en el mundo es desigual en dos aspectos, uno de ellos en la concepción teórica del Proceso de Enfermería y en su implementación en el plano clínico ${ }^{6}$, confirmando este hecho Ledesma $\mathrm{D}^{7}$, opina que el $P E$ se ha incorporado como eje metodológico que guía la enseñanza teórica/práctica de estudiantes en la mayoría de las escuelas y Facultades de Enfermería en México.

Ledesma $\mathrm{D}$, realizó un estudio de investigación donde reporta que sólo el 78\% de los estudiantes tienen conocimiento del PE pero sólo el 66.5\% lo aplican. La etapa de evaluación representa el menor porcentaje de conocimiento (68.5\%), y la etapa de menor aplicación es la de planeación (54.3\%) lo que explica que los factores que influyen en estas dos variables son poco tiempo de práctica y permanencia en los servicios clínicos, lo que reduce la implementación del proceso en los ámbitos de práctica asistencial, sin perder de vista que existe también una gran diversidad de criterios para la enseñanza-evaluación del
PE y la resistencia del estudiante para utilizarlo en sus actividades del área clínica .

Por lo tanto, los programas de enfermería contemplan el PE, como método fundamental para desarrollar los contenidos teóricos y prácticos. Aún cuando el $\mathrm{PE}$ es un contenido invariante dentro de la currículo de enfermería los egresados de enfermería presentan dificultades para aplicar este conocimiento adquirido durante su formación, el cual se evidencia al ingresar como trabajadores a las instituciones de salud donde lo aplican escasas veces en la atención del paciente.

Hoy las instituciones de salud pública y privadas están respondiendo a las exigencias sociales y haciendo esfuerzos por aplicar el PE en sus actividades diarias. Pero la brecha entre demandas ideales de los servicios de salud y la realidad dentro de la rutina de la atención de enfermería es muy distinta.

Por lo anterior nos da una visión general sobre la trascendencia de esta metodología científica y su relevancia en el cuidado del paciente en los ámbitos de actuación de enfermería.

\section{MATERIAL Y MÉTODOS}

Se realizó un estudio descriptivo, retrospectivo, y transversal. Las variables de estudio fueron: nivel de conocimientos respecto al PE, percepción de autoeficacia en la aplicación del PE y el papel del profesor en la enseñanza del PE.

Se selecciono una muestra a conveniencia de los alumnos del tercer año de la carrera de Licenciatura en Enfermería.

La muestra piloto se aplicó a cinco alumnos que cursaron el tercer año de la carrera de licenciatura en enfermería con la finalidad de aumentar la confiabilidad al instrumento donde se hicieron las modificaciones sugeridas en relación a redacción de algunos ítems.

Se utilizó un cuestionario para medir el nivel de conocimientos acerca de la aplicación del PE y percepción de autoeficacia, elaborado por Bernal Martha y Ponce Gandhy ${ }^{9}$, con una confiabilidad 0.87. Conformado por preguntas abiertas y cerradas, que consta de 3 partes: la primera solicita datos sociodemográficos, que incluye edad, sexo, promedio general, semestre que cursa y condición laboral; la segunda identifica el nivel de conocimientos del PE a través de 15 preguntas de opción múltiple y la tercera identifica a través de 28 preguntas tipo likert, con valores de 5 a 1 , que miden su eficacia para la aplicación del PE. Para este estudio, se realizó el análisis e interpretación de los datos, utilizando programas como SPSS para el manejo de estadística descriptiva. 


\section{RESULTADOS}

El grupo de investigación incluyó 54 alumnos del VI semestre de licenciatura en enfermería, donde la media de la edad se encuentra en 21 años. De los cuales el 75.9\% de la población estudiada no trabaja y un $92.6 \%$ es del género femenino.

El 64.8\% de la muestra cuenta con un nivel de conocimientos suficientes sobre el PE de los cuales la media fue calificación fue de 8.3.

En la revisión teórica del PE en el aula el 25.9\% de los alumnos expresan que presentaron dificultad en la etapa de planeación, un $24.0 \%$ en la valoración, otro porcentaje similar (24.0\%) en la fase diagnóstica, el 7.4\% en la ejecución y un 5.5\% en todas las etapas del PE.

En relación a la aplicación del PE en la práctica clínica, el $24.0 \%$ de los alumnos presentaron dificultades en la etapa de planeación, un $22.2 \%$ de los alumnos en evaluación, 16.6\% en la ejecución, un $14.8 \%$ en la valoración, y un $9.2 \%$ en la fase diagnóstica.

El 63\% de los alumnos expresan que los docentes facilitaron el aprendizaje del PE y el 35.2\% refiere que requirió ayuda de un compañero para aplicar el PE en el área hospitalaria.

Los alumnos consideran que una estratégia eficaz para aprender el PE es a través de casos 48.1\%, mientras que el 27.8 comenta que a través de la práctica con el paciente, el 16.7\% es con ayuda de sus compañeros como monitores y sólo un $2 \%$ manifiesta que el personal de enfermería contribuyo en la enseñanza del PE.

El 40.7 de los alumnos comentan que siempre aplicaron el PE en la práctica hospitalaria mientras que el 37\% casí siempre lo aplicaron y el $16.7 \%$ algunas veces.

El 81.4\% de los alumnos comenta que aplicaron el PE en uno a dos pacientes por día, mientras que el $9.2 \%$ lo aplicaron en tres o cuatro pacientes durante el día y otro $9.2 \%$ no contestó este cuestionamiento.

Los factores principales que obstaculizan la aplicación del PE fueron según el 53.7\%, falta de tiempo para realizar esta metodología en los ámbitos de práctica hospitalaria, el 20.3\% expresa que es por exceso de pacientes y falta de tiempo, el 9.2\% refiere es por la falta de conocimiento, y el 5.5\% menciona que fue por falta de tiempo y conocimientos y un porcentaje similar expreso que es por exceso de pacientes (5.5\%).

En relación a la autoeficacia para aplicar el PE el 50\% de los alumnos expresan que se sienten seguros de aplicarlo mientras que el $38.9 \%$ esta relativamente seguro.
El 50\% de los alumnos opinan que están seguros que el PE les permite resolver problemas del paciente; en tanto el 20.4\% dicen estar completamente seguros y 20.4\% están relativamente seguros.

El 77.8\% de los alumnos expresan que siempre les ayuda ver al profesor realizar el PE en el área hospitalaria; mientras que un $16.7 \%$ dice que casí siempre. Por lo tanto, el $77.8 \%$ de los alumnos refiere que el profesor hace énfasis con frecuencia en la utilización del PE en el área de práctica y el 16.7\% menciona que lo hace casí siempre.

El 27.8\% de los estudiantes expresan que casí siempre el personal de enfermería solicita al alumno realizar el PE mientras que el $16.7 \%$ dicen que lo hacen siempre y el 14.8\% manifiesta que casí nunca y el 11.1\% refieren que nunca lo hacen.

Un $46.3 \%$ de los alumnos comentan que el profesor siempre refuerza los logros del alumno al aplicar el proceso; el 35.2\% manifiesta que lo hace casí siempre y el $16.7 \%$ menciona que el profesor refuerza algunas veces está actividad en los estudiantes.

El 58.2\% de los alumnos manifiestan que el paciente se encuentra satisfecho con los cuidados otorgados por el estudiante, mientras que el 37\% comenta que está medianamente satisfecho y el 3.7\% no contesto.

\section{DISCUSIÓN}

Los conocimientos sobre el proceso son suficientes en los alumnos del VI semestre de la carrera de licenciatura en enfermería y la autopercepción de autoeficacia; refieren que se sienten seguros de aplicarlo ante situaciones reales según el 50\% y relativamente seguros el 38.9\%.

A diferencia del estudio de Ledesma donde encuentra que los estudiantes tienen conocimiento del PE pero sólo el 65\% lo aplican ${ }^{7}$.

Ledesma manifiesta también que los estudiantes tienen conocimiento sobre el PE y en cada una de sus etapas pero; lo aplican en menor proporción, esto indica dificultades en la integración teórico-práctica que deberán ser consideradas para fortalecer el proceso formativo ${ }^{7}$.

A diferencia de lo que señala Bernal 2008; en la investigación relacionada al nivel de conocimientos acerca del PE y percepción de autoeficacia para su aplicación, comenta que el nivel de conocimientos sobre el proceso en los semestres intermedios de la carrera de licenciatura era de dominio amplio y claro donde los estudiantes consideran fácil de aplicar el PE y es alto su nivel de autoeficacia ${ }^{8}$. 
En relación a la aplicación del PE en la práctica clínica presentaron dificultades el $24.0 \%$ de los alumnos en etapa de planeación y un $22.2 \%$ de alumnos en evaluación y 16.6\% en la fase de ejecución, un $14.8 \%$ en la fase de valoración, y un 9.2\% en la fase diagnóstica del PE.

A diferencia del estudio de Ledesma; sobre conocimiento y aplicación del PE por estudiantes de licenciatura se encontró que la etapa de evaluación, representa en un menor porcentaje de conocimiento (68.5\%) y la etapa de menor aplicación es la planeación (54.3) y expresa que los factores que influyen para la no aplicación son poco tiempo de práctica y permanencia en los servicios, así como la diversidad de criterios para su enseñanza y evaluación, y la no disposición del estudiante para utilizarlo 7 . Porque en la práctica los alumnos se preocupan por integrarse al trabajo rutinario de las enfermeras y no aplican los conocimientos a situaciones particulares ${ }^{9}$.

El 50\% de la población estudiada refiere que está seguro que el PE ayuda a resolver problemas, en tanto el 20.4\% dicen estar completamente seguros y $20.4 \%$ están relativamente seguros.

Además; el 88.8\% de los alumnos expresan que los pacientes se muestran satisfechos con el cuidado proporcionado a través del PE; por lo tanto, se muestran más accesibles, amables, y la comunicación es más fluída, por lo que coinciden con Jiménez 2007, que refiere que el uso del PE en la práctica profesional facilita el cuidado integral a los usuarios.

Los trabajos realizados por Andrade, Loza, García, Tovar, Acosta, Morales y Jiménez, lo apoyan y afirman que el PE aumenta la calidad de los cuidados integrales que aumenta la eficacia y eficiencia en la atención de enfermería, proporcionando uniformidad en los cuidados y aumenta la posibilidad de evaluar la calidad de la atención de enfermería ${ }^{10-16}$.

El 79.6\% de los alumnos expresan que el PE es útil para el aprendizaje. Lo que concuerda con Aguilar 2004 ${ }^{9}$, que refiere que el PE constituye la columna vertebral en la formación de la enfermería profesional.

El 63\% de los alumnos manifiestan que los docentes facilitaron el aprendizaje del PE y el 35\% comenta que fue con ayuda de los compañeros. Ledesma manifiesta que existe diversidad de criterios para su enseñanza y evaluación.

Los alumnos expresan que en el aula las estrategías didácticas favorecen el aprendizaje de este contenido; a través de casos según el 48.1\% con la práctica clínica de acuerdo con el 27.8\%, y con los compañeros como lo menciona el $16.7 \%$ de los informantes.

González Halcones, manifiesta y precisa que la aplicación de pruebas situacionales es el sistema más útil para evaluar el aprendizaje, porque tienen como objeto medir el grado en que los formados son capaces de poner en práctica conocimientos, habilidades y actitudes enseñadas en el transcurso de la acción formativa. Para ello, se recrea una situación que simula las condiciones y requisitos que plantea la actividad laboral real ${ }^{17}$.

\section{CONCLUSIONES}

El nivel de conocimientos en el alumno es suficiente para la aplicación del PE en el área hospitalaria; pero existen diversos factores que intervienen en la aplicación para el cuidado del paciente y enseñanza-aprendizaje en las áreas hospitalarias.

Los factores que favorecen la enseñanza-aprendizaje son: estrategías didácticas utilizadas por los docentes en el tema los cuales ayudaron a los alumnos apropiarse de este contenido y el tiempo de práctica clínica fue suficiente para el aplicar el PE.

Los contenidos de enseñanza-aprendizaje sobre el PE utilizados por los maestros; tanto en la teoría como en la práctica son adecuados y útiles para ayudar al alumno a aprender.

Los factores que obstaculizan la enseñanza-aprendizaje del PE son: percepción del alumno sobre la autoeficacia en la aplicación del PE que varía según la personalidad del alumno, lo que origina actitudes que limitan o favorecen la realización de intervenciones enfermeras de acuerdo al problema enfermero identificado en el paciente, otras causas que limitaron la aplicación del PE fue la falta de tiempo en las áreas hospitalarias y exceso de pacientes. Se deduce que la efectividad en el aprendizaje del PE aún no logra el efecto esperado en las áreas hospitalarias.

\section{RECOMENDACIONES}

1. Que el profesor utilice los casos clínicos y se apoye en alumnos que funjan como monitores para favorecer el aprendizaje del PE

2. Proporcionar y demostrar modelos de reforzamiento positivos del PE; para ayudar al entendimiento y enseñanza del PE.

3. Planear y organizar actividades en que el alumno aplique el PE.

\section{REFERENCIAS}

1. Salas Perea Ramón S. La calidad en el desarrollo profesional: avances y desafíos. Salasram@infomed. sld. cu. www.bvs. sld.cu/ revistas/ ems/ vol14_2_00 /ems03200.htm

2. Iyer P, Taptich B, Bernocchi D. Proceso y diagnóstico de enfermería. $3^{\circ}$ ed. México Mc Graw-Hill Interamericana 1997.

3. Stephen Covey. Los siete hábitos de las personas altamente efectivas. Barcelona. Ediciones Paidós. 1989. 
4. Koonz H. Administración una perspectiva global. $12^{\text {a }}$ ed. Madrid Interamericana. Mc Graw-Hill. 2004.

5. Robbins S. Administración. 8a ed. Pearson Educación. 2005.

6. Hdz. V. Proceso de enfermería: su teoría y su práctica. Desarrollo Científico en Enfermería 2006;14(7):274-8.

7. Ledesma M. Conocimiento y aplicación del proceso de enfermería por estudiantes de licenciatura. Desarrollo Científico en Enfermería 2005;13(3):82-6.

8. Bernal M, Ponce G. Nivel de conocimientos del proceso enfermero y la percepción de autoeficiencia para su aplicación. Rev Enfermería Inst Méx Seguro Soc 2008,16(1):3-6.

9. Morales A. Perspectiva del proceso de enfermería. Desarrollo Científico en Enfermería 2006;14:(8):321-5.

10. Andrade R. Proceso enfermero para brindar atención de calidad. Desarrollo Científico en Enfermería 2004;(12):8:17-9.

11. Loza H. Impacto de la implementación del proceso de atención de enfermería en el INNN MVS, mediante una prueba piloto. Enferm Neurológica (Méx.) 2005;4:(1):1-6.

12. García G. Aportaciones de las taxonomías al proceso de enfermería. Desarrollo Científico en Enfermería 2006;14:(3):92-8.

13. Tovar M. Proceso de cuidado de enfermería a paciente con amenaza de parto prematuro. Desarrollo Científico en Enfermería 2006; (8):316-20.

14. Acosta L. Proceso de enfermería a paciente posoperada de persistencia de conducto arterioso. Desarrollo Científico en Enfermería 2007;15:(1):27-35.

15. Morales A. Epistemología del proceso de enfermería. Desarrollo Científico en Enfermería 2007;15:(2):84-8.

16. Jiménez N. Proceso de enfermería a paciente con preeclampsia severa. Desarrollo Científico en Enfermería 2007;15:(9):410-5.

17. La evaluación del proceso de enseñanza-aprendizaje. Fundamentos básicos. Miguel Ángel González Halcones. www.uclm.es/profesorado/ ricardo/docencia_e_investigacion/4/EVALUACION_Halcones.doc 